\title{
Model reduction of a direct spring-loaded pressure relief valve with upstream pipe
}

\author{
Csaba Hős and Csaba Bazsó \\ Department of Hydrodynamic Systems, Budapest University of Technology and Economics, \\ Budapest, Hungary \\ csaba.hos@hds.bme.hu csaba.bazso@hds.bme.hu \\ AND \\ Alan Champneys* \\ Department of Engineering Mathematics, University of Bristol, Bristol, UK \\ *Corresponding author: a.r.champneys@bristol.ac.uk
}

[Received on 23 October 2013; revised on 29 May 2014; accepted on 12 June 2014]

\begin{abstract}
A mathematical model is developed of a spring-loaded pressure relief valve connected to a reservoir of compressible fluid via a single, straight pipe. The valve is modelled using Newtonian mechanics, under assumptions that the reservoir pressure is sufficient to ensure choked flow conditions. The usual assumptions of ideal gas theory lead to a system of first-order partial differential equations for the motion, energy and momentum of the fluid in the pipe.

A reduced-order model is derived using a collocation method under the assumption that the dominant pipe dynamics corresponds to a standing quarter wave. The model comprises just five non-linear ordinary differential equations, representing the position and velocity of the valve body, the pressure in the tank and the velocity and pressure amplitudes of the pipe quarter wave. Through comparison with simulations of the full model using a Lax-Wendroff method, it is shown that the reduced model is quantitatively accurate and is able to predict the onset of an oscillatory valve-chatter instability. The basic trends of this instability are shown to be robust to the inclusion of pipe friction and convective effects.
\end{abstract}

Keywords: pressure relief valve; valve chatter; valve instability; gas dynamics.

\section{Introduction}

Pressure relief valves (PRVs) are essential elements of pressurized systems such as hydraulic power transmission circuits, natural gas systems or chemical plants. They prevent the pressure level from increasing above a prescribed set pressure by releasing an excess of fluid. The simplest and most robust type of PRVs are direct spring-loaded valves, which, due to their simplicity and reliability, are often employed in safety-critical applications.

This study focuses on the dynamics of such conventional direct spring-loaded PRVs assembled with a simple disc plate, the so-called poppet valves. These PRVs are mounted to the protected reservoir either directly or more commonly_due to practical installation or operational considerations — via an attendant upstream pipeline. However, the presence of the connecting pipeline might lead to a risky combination of the valve body dynamics, pipeline dynamics and the pressure dynamics of the reservoir. Due to the complex fluid-structure interaction, such systems have a tendency to self-oscillate and such dangerous oscillations are challenging to predict in the design phase. 
The rapid increase of the computational resources and the development of computational fluid dynamics (CFD) techniques provide a great potential for analysing distributed parameter systems (see, e.g. Viel, 2011). However, performing quick, parametric and qualitative studies with such techniques are nearly impossible even nowadays due to the large computational effort needed for time-dependent CFD runs. The aim of the present study is to derive a hydro-mechanical model of the reservoir-pipePRV system that consists of only ordinary differential equations (ODEs) (allowing parametric stability analysis), yet which captures the essence of the dynamics close to the stability loss and hence is suitable for predicting the onset of self-excited oscillations already during the design phase.

A considerable amount of literature has been published on the description and analysis of valve systems. In 1973, Green \& Woods (1973) provided the first comprehensive discussion of the possible causes of valve instabilities, suggesting that they can be induced as a result of five different effects: the interaction between the poppet and other elements, flow transition from laminar to turbulent during opening and closing, a negative restoring force, hysteresis of the fluid force and fluctuating supply pressure. This paper shall focus on the first of these, specifically instability due to interaction between the poppet and an inlet pipe (although, as we shall see, this can also be interpreted as an effective negative restoring force on the valve provided by an acoustic wave).

Instabilities due to the other four effects identified by Green and Woods have been analysed by a number of other authors, see, for example, Kasai (1968), McCloy \& McGuigan (1964), Madea (1970a,b), Nayfeh \& Bouguerra (1990), Vaughan et al. (1992), Moussou et al. (2010), Beune (2009) and Song et al. (2013). Conventional PRVs subject to built-up back pressure have also been widely investigated, see, e.g. Francis \& Betts (1998), Chabane et al. (2009) and Moussou et al. (2010). In this paper, we do not consider effects downstream piping. Oscillations in other valve systems have also been studied, e.g. in plug valves (D’Netto \& Weaver, 1987), compressor valves (Habing \& Peters, 2006), ball valves (Nayfeh \& Bouguerra, 1990), pilot-operated two-stage valves (Botros et al., 1997; Zung \& Perng, 2002; Ye \& Chen, 2009) and control valves (Misra et al., 2002). Again, such studies go beyond the scope of the present work.

The first serious discussion of self-excited instabilities of poppet valves emerged in the 1960s. Funk (1964) in 1964 discussed the influence of valve chamber volume and pipe length within a hydraulic circuit on the stability of a poppet valve. He found that such valves are inclined to become unstable at a critical frequency that coincides with the fundamental vibratory mode of the pipeline. Moreover, the severity of the instability increases with the length of the pipe. In 1968, Kasai (1968) developed this analysis by deriving equations of motion for such a poppet valve and inlet piping system. Based on linear stability analysis, he established formulae for predicting instability in the valve. The results were shown to be in broad agreement with experiments. A similar configuration was studied by Thomann (1976) who found that the valve motion can couple to the acoustic oscillation of the pipe, leading to amplified oscillation of the system. He also developed analytical criteria for the loss of stability, finding good agreement with experiments.

Later, MacLeod (1985) developed a model that includes gas dynamical issues such as choked flow capable of predicting the region of stable operation of a simple spring-loaded PRV mounted directly onto a gas-filled pressure vessel. Then, in the 1990s, Hayashi (1995) and Hayashi et al. (1997) carried out detailed linear and global stability analyses of a poppet valve circuit and represented examples of 'soft' and 'hard' self-excited vibration. They revealed that for the same conditions several pipe vibration modes can become simultaneously unstable, with the number of unstable modes increasing with pipe length. An interesting outcome of this study was the discovery that self-excited valve vibrations can occur even below the set pressure, when ordinarily the valve would not be expected to open. This result coincides with one obtained by Botros et al. (1997) which states that for higher values of the pipe length 
two modes evolve in the system while for lower values of the pipe length the vibration is primarily in the fundamental, quarter-wave model (QWM). They also found that maximum amplitude occurs when the oscillation frequency coincides with the quarter-wave natural frequency; for lower and higher values of the pipe length the amplitude decreases.

In early work by two of us together with Licsko et al. (2009), we used non-linear dynamical system methods to analyse a low-order system of ODEs describing a simplified version of the set up in Kasai (1968) and Hayashi et al. (1997), ignoring the effect of the pipe. Here, we showed that upon reduction of the inlet flow rate, loss of stability is due to a Hopf bifurcation. The system was further investigated further by us in Hős \& Champneys (2012), where we elucidated the nature of further grazing bifurcations in the system that underlie impacting motion between the valve and its seat, and performed detailed two-parameter continuation. At the same time, in Bazsó \& Hős (2013), we report experimental results in a hydraulic system that showed qualitative agreement to the non-linear dynamics predicted in Licsko et al. (2009). That paper also presented a preliminary stability map that shows the frequency of the evolving self-excited vibration along the boundary of loss of stability. The purpose of the present paper is to derive an extension to the reduced-order model in Licsko et al. (2009) to include the more realistic effect of a downstream inlet pipe that is capable of correctly capturing the onset of instability.

The rest of this paper is outlined as follows. Section 2 defines the physical model we propose to study, and derives a complete formulation of its dynamics using the usual assumptions of compressible fluid flow within the pipe. A straightforward numerical solution method is described using the LaxWendroff method. Section 3 then considers model reduction close to instability by assuming the pipe acoustic energy is contained within the first mode. Section 4 presents a comparison between numerical solutions of the full and reduced model and shows good agreement for the prediction of the onset of the initial instability. Finally, Section 5 draws conclusions.

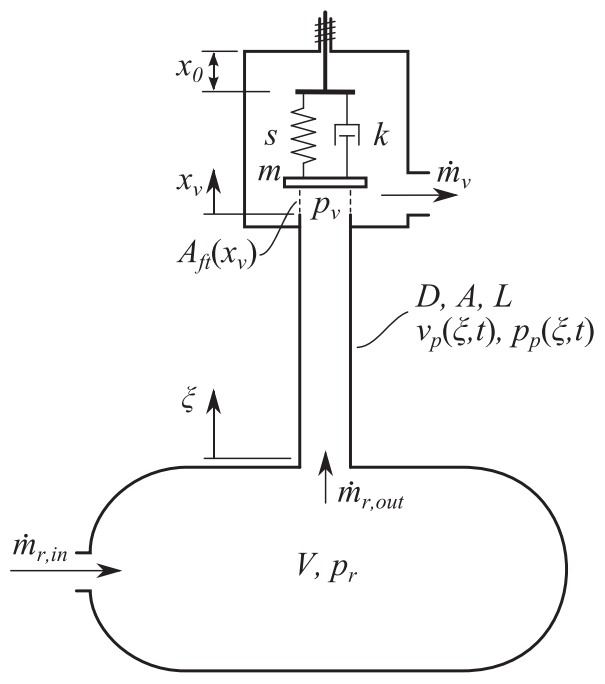

FIG. 1. Mechanical model of the system. 


\section{Mathematical modelling}

Consider the system depicted in Fig. 1 consisting of a reservoir, a pipe and a direct spring-loaded valve. The reservoir is taken to be perfectly rigid. The mass flow rate $\dot{m}_{r \text {,in }}$ of the compressible fluid entering the reservoir is presumed either to be constant or to vary slowly when compared with other timescales present in the system. The change of state in the reservoir is assumed to be isentropic, that is, there is no heat exchange with the surroundings and there are no internal losses. The mass outflow from the reservoir $\dot{m}_{r \text {,out }}$ is in general assumed to be time varying.

The flow in the long, thin pipe is assumed to be described by 1D unsteady gas-dynamics theory, including the effects of wall friction. Such an approach captures the inertia of the fluid, its compressibility and pressure losses, which allows for the presence of both wave effects and damping.

The valve body is modelled as a single degree-of-freedom oscillator that obeys a Newtonian equation of motion. We assume ambient backpressure behind the valve and, due to the relatively high pressure difference between the end of the pipe and the surroundings, the flow through the valve will be assumed to be choked. That is, the fluid velocity reaches the local sonic velocity at the vena contracta and hence backpressure does not affect upstream flow.

\subsection{Valve body dynamics}

The valve itself consists of an inertial mass, the valve body and a pre-compressed spring. The motion of such PRVs is usually very weakly damped. This is because international safety standards dictate that if the pressure upstream of the valve reaches the set pressure-that which is sufficient to overcome the spring pre-compression - then the valve must open as quickly as possible. Nevertheless, we shall include some viscous damping in the model to represent the internal damping of the spring and the drag and added-mass effect of the fluid (see, e.g. Khalak \& Williamson, 1997; Askari et al., 2013). The motion of the valve body can be described as a single degree-of-freedom rigid body, with mass $m$, spring constant of $s$ and viscous damping of $k$. The pre-compression of the spring will be denoted by $x_{0}$ while $x_{v}$ stands for the displacement of the valve body. The governing equation is thus given by

$$
m \ddot{x}_{v}+k \dot{x}_{v}+s\left(x_{0}+x_{v}\right)=F_{\text {fluid }} \text { for } x_{v}>0,
$$

where $F_{\text {fluid }}$ is the force on the valve due to the fluid. Once the valve body hits the seat $\left(x_{v}=0\right)$, we apply the impact law

$$
v_{v}^{+}=-r v_{v}^{-},
$$

with $v_{v}^{+}$and $v_{v}^{-}$being the velocities immediately before and after the impact and $r$ stands for the restitution coefficient.

The fluid force on the valve can be decomposed into two parts; from the pressure difference between the ambient pressure $p_{0}=0$ and the fluid pressure $p_{v}$ and from the momentum of the fluid:

$$
F_{\text {fluid }}=F_{\text {pressure }}+F_{\text {momentum }}=\left(p_{v}-p_{0}\right) A+\dot{m} v=\left(p_{v}-p_{0}\right) A+\frac{\dot{m}^{2}}{\rho_{v} A},
$$

where $A$ is the valve area on which the pressure acts, which for simplicity we take to be equal to the cross-sectional area of the pipe, independent of the valve lift, $\rho_{v}$ is the density of the fluid entering the valve, $\dot{m}$ and $v$ are the mass flow rate and the velocity of the fluid at the valve, respectively. The above expression also assumes purely radial exit jet. Other fluid forces such as, e.g. the drag due to the wall shear stress are neglected. 
TABLE 1 Ratio of the momentum and pressure force defined by (3) for several valve openings. The parameter values are given in Table 2.

\begin{tabular}{lcc}
\hline$x_{v} / x_{\max }(\%)$ & $\dot{m}(\mathrm{~kg} / \mathrm{s})$ & $F_{\text {momentum }} / F_{\text {fluid }}(\%)$ \\
\hline 20 & 1.31 & 1.08 \\
40 & 2.72 & 4.19 \\
60 & 4.12 & 8.96 \\
80 & 5.46 & 14.9 \\
100 & 6.66 & 21.5 \\
\hline
\end{tabular}

For the sake of simplicity, in what follows we assume that the fluid force derives only from the pressure, i.e. $F_{\text {momentum }} \ll F_{\text {pressure }}$ in (3). To evaluate this assumption, we compute the actual values of the force components for a particular valve, whose parameters are given in Table 2. The results are shown in Table 1, revealing that at maximum valve lift the momentum force is $21.5 \%$ of the fluid force and the error is beneath $10 \%$ if the relative lift is below $70 \%$. The contribution of the momentum force to the global dynamics is not the primary focus of the present work, however, it can be easily incorporated in the model derived later. For more details on the effect of this force component, we refer the reader to Francis \& Betts (1998).

Assuming that the valve is closed $x_{v}=0$, so that no flow is present in the pipe (thus $p_{r}=p_{v}$ ), then the reservoir pressure at which the valve opens is referred to as the set pressure and is given by

$$
p_{\text {set }}=\frac{s x_{0}}{A}-p_{0}
$$

\subsection{Reservoir pressure dynamics and orifice flows}

When modelling the fluid dynamics in the orifice and the reservoir, we should keep in mind that we are trying to capture the behaviour of PRVs. Such valves are only designed to open when there is a significant pressure difference between the upstream pressure $p_{v}$ and the ambient, downstream, pressure $p_{0}$. It is therefore reasonable to assume that this difference is large enough for choked flow to occur. This means that flow reaches the sonic velocity at the narrowest cross-section, the so-called vena contracta and hence the downstream pressure does not affect the flow rate (for details, see Zucrow \& Hoffman, 1976). As an illustration to quantify when this assumption is valid, for the case of air whose specific heat ratio is $\kappa=c_{p} / c_{v}=1.4$, choked flow occurs if the pressure ratio reaches (see also Zucrow \& Hoffman, 1976)

$$
\frac{p_{v}}{p_{0}} \geqslant\left.\left(\frac{\kappa+1}{2}\right)^{(\kappa /(\kappa-1))}\right|_{\kappa=1.4} \geqslant 1.8929 .
$$

Now consider choked flow through an orifice with discharge coefficient $C_{d}$, absolute upstream pressure and density $p_{v}$ and $\rho_{v}$, absolute downstream pressure $p_{0}$ and orifice flow-through area $A_{f t}$. This area is defined as the cross-sectional area of the vena contracta. Most valves are designed such that

$$
A_{f t} / A \ll 1 .
$$

We assume a simple topology as in Fig. 1, so that the flow-through area is represented by a small cylindrical surface between the seat and the valve disc, which can be expressed as a simple function of 
the valve lift:

$$
A_{f t}\left(x_{v}\right)=D \pi x_{v}:=c_{1} x_{v}
$$

The mass flow rate for choked, compressible flow can be expressed as (see, e.g. Zucrow \& Hoffman, 1976)

$$
\dot{m}=C_{d} A_{f t}\left(x_{v}\right) c_{2} \sqrt{\rho_{v} p_{v}},
$$

where the discharge coefficient $C_{d}$ is an empirically derived constant that describes velocity nozzle loss as the fluid is vented to the atmosphere and the constant $c_{2}$ is defined via

$$
c_{2}=\sqrt{\kappa\left(\frac{2}{\kappa+1}\right)^{(\kappa+1) /(\kappa-1)}},
$$

with $\kappa$ being the heat capacity ratio. If, for convenience, we collect the constants into a single term $\zeta=c_{1} c_{2} C_{d}$, we get

$$
\dot{m}=\zeta x_{v} \sqrt{p_{v} \rho_{v}}
$$

The imbalance between the constant inflow and outflow rates, $\dot{m}_{r \text {, in }}$ and $\dot{m}_{r \text {,out }}$, results in changes in the reservoir pressure. We assume that the fluid obeys the ideal gas law, $p / \rho=R T$, and that the process in the reservoir is isentropic, $p / \rho^{\kappa}=$ constant. Mass balance in the reservoir of volume $V$ therefore gives

$$
\frac{\mathrm{d} m}{\mathrm{~d} t}=V \frac{\mathrm{d}}{\mathrm{d} t}\left(\rho_{r}(t)\right)=V \frac{\mathrm{d}}{\mathrm{d} t}\left(\frac{p(t)}{R T(t)}\right)=\dot{m}_{\text {in }}-\dot{m}_{\text {out }} .
$$

Given an ambient reference state $p_{0}, T_{0}$, the temperature can be related to the pressure via

$$
T(t)=T_{0}\left(\frac{p_{r}(t)}{p_{0}}\right)^{(\kappa-1) / \kappa},
$$

which gives

$$
\dot{p}_{r}=\kappa \frac{R T_{0}}{V}\left(\frac{p_{r}}{p_{0}}\right)^{(\kappa-1) / \kappa}\left(\dot{m}_{\text {in }}-\dot{m}_{\text {out }}\right)=\frac{a^{2}}{V}\left(\dot{m}_{\text {in }}-\dot{m}_{\text {out }}\right),
$$

with $a=\sqrt{\kappa R T}$ being the sonic velocity. We emphasize again that the sonic velocity in the reservoir depends on temperature, which—as described by (9)—changes with the reservoir pressure.

\subsection{Pipeline dynamics}

The flow inside the pipe is assumed to be compressible and effectively 1D. We also allow for wall friction, which will result in pressure loss. The gas is assumed to be ideal but the change of state is not fixed (i.e. it can be isentropic, isothermic, etc.) hence, besides equations of continuity and momentum balance, we also need to solve an energy-balance equation. We assume that the pipe cross-section $A=$ const. and make an adiabatic pipe approximation (i.e. no heat flux through the wall). Under these 
assumptions, the gas dynamical governing equations can be written in the compact form

$$
\frac{\partial \mathcal{U}}{\partial t}+\frac{\partial \mathcal{F}}{\partial \xi}=\mathcal{Q}
$$

with

$$
\mathcal{U}=\left(\begin{array}{c}
\rho \\
\rho v \\
\rho e
\end{array}\right), \quad \mathcal{F}=\left(\begin{array}{c}
\rho v \\
\rho v^{2}+p \\
\rho e v+p v
\end{array}\right) \quad \text { and } \quad \mathcal{Q}=\left(\begin{array}{c}
0 \\
F_{s} \\
0
\end{array}\right)
$$

Here, $\xi$ denotes the axial coordinate along the pipe. The unknown dependent variables are density $\rho(\xi, t)$, velocity $v(\xi, t)$, pressure $p(\xi, t)$ and energy $e(\xi, t)$.

Now, the overall energy of the gas comprises the sum of its internal energy $c_{v} T$ and kinetic energy $v^{2} / 2$ :

$$
e(\xi, t)=c_{v} T(\xi, t)+\frac{v(\xi, t)^{2}}{2}
$$

and, using the ideal gas law we have

$$
p(\xi, t)=\rho R T(\xi, t)
$$

Hence, using these two equations, pressure and temperature can be expressed in terms of the dynamic unknowns $v$ and $e$. The source term on the right-hand side of (11) is due to the pipe friction, and can be expressed as

$$
F_{s}=\frac{\rho \lambda v|v|}{2 D}
$$

with $\lambda$ being the pipe friction coefficient and $D$ the pipe (inner) diameter.

The boundary conditions are defined as follows. At $\xi=0$, we assume isentropic inflow into the pipe. That is, the total enthalpy at the reservoir $\left(h_{r}=c_{p} T_{r}\right)$ and at the pipe entrance are equal:

$$
c_{p} T_{r}(t)=c_{p} T(0, t)+\frac{1}{2}(v(0, t))^{2},
$$

which can also be written is terms of pressure, because

$$
\frac{T_{r}}{T_{0}}=\left(\frac{p_{r}}{p_{0}}\right)^{z} \quad \text { and } \quad \frac{T(0, t)}{T_{0}}=\left(\frac{p(0, t)}{p_{0}}\right)^{z}, \quad \text { with } z=(\kappa-1) / \kappa .
$$

At $\xi=L$, we required that the mass flow rate leaving the valve is equal to the mass flow rate within the valve, see (8), thus we have

$$
\rho(L, t) A v(L, t)=\zeta x_{v} \sqrt{\rho(L, t) p(L, t)} .
$$

Equations (11-14) represent a well-posed initial-value problem first-order hyperbolic system of partial differential equations. We refer to this as the full gas dynamical model (GDM).

\subsection{Numerical solution technique}

We have solved the GDM using a standard two-step Lax-Wendroff method, as described in Cebeci et al. (2005) and Warren (1983). This is a second-order finite difference scheme of predictor-corrector type. 
Here, we briefly recall the main steps. Let $i$ and $j$ denote the equidistant spatial and temporal grid points, respectively,

$$
\mathcal{U}_{j}^{i}=\mathcal{U}\left(\xi_{i}, t_{j}\right),
$$

with temporal resolution $\Delta t$ and spatial resolution $\Delta \xi$. The method is comprised of several steps which are set out as follows:

1. Advance $\mathcal{U}_{j+1 / 2}^{i+1 / 2}$ at the half-time level and middle grid points from

$$
\frac{\mathcal{U}_{j+1 / 2}^{i+1 / 2}-\mathcal{U}_{j+1 / 2}^{i}}{\Delta t / 2}+\frac{\mathcal{F}_{j+1}^{i}-\mathcal{F}_{j}^{i}}{\Delta \xi}=\frac{\mathcal{Q}_{j+1}^{i}+\mathcal{Q}_{j}^{i}}{2} \quad \text { where } \mathcal{U}_{j+1 / 2}^{i}=\frac{\mathcal{U}_{j+1}^{i}+\mathcal{U}_{j}^{i}}{2}
$$

2. Compute the primitive variables $\left(p, \rho, v\right.$ and $e$ ) and find the fluxes $\mathcal{F}_{j+1 / 2}^{i+1 / 2}$.

3. Take a full time step to compute $\mathcal{U}_{j}^{i+1}$ with the help of the centred fluxes $\mathcal{F}_{j+1 / 2}^{i+1 / 2}$ :

$$
\frac{\mathcal{U}_{j}^{i+1}-\mathcal{U}_{j}^{i}}{\Delta t}+\frac{\mathcal{F}_{j+1 / 2}^{i+1 / 2}-\mathcal{F}_{j-1 / 2}^{i+1 / 2}}{\Delta \xi}=\frac{\mathcal{Q}_{j+1 / 2}^{i+1 / 2}+\mathcal{Q}_{j-1 / 2}^{i+1 / 2}}{2} .
$$

For stability, the time step $\Delta t$ should be small enough to ensure that the 'information' propagating with velocity $a+|v|$ does not 'jump over' a cell. That is,

$$
\Delta t<C \min _{j}\left(\frac{\Delta \xi}{a_{j}+\left|v_{j}\right|}\right)
$$

where $C<1$ is the safety factor known as the Courrant number and $a_{j}=\sqrt{\kappa R T_{j}}$.

Boundary conditions are implemented using the method of isentropic characteristics, see Zucrow \& Hoffman (1976), Cebeci et al. (2005) and Warren (1983) for a detailed explanation. Essentially,

TABLE 2 Parameter values used in the computations.

\begin{tabular}{lcc}
\hline Quantity & Symbol & Value \\
\hline Mass flow rate & $\dot{m}_{r, \text { in }}$ & $0-10 \mathrm{~kg} / \mathrm{s}$ \\
Pipe length & $L$ & $0-2 \mathrm{~m}$ \\
Pipe diameter & $D$ & $50 \mathrm{~mm}$ \\
Reservoir volume & $V$ & $10 \mathrm{~m}^{3}$ \\
Mass of valve body & $m$ & $1.5 \mathrm{~kg}$ \\
Spring constant & $s$ & $125 \mathrm{kN} / \mathrm{m}$ \\
Damping coefficient & $k$ & $20 \mathrm{Ns} / \mathrm{m}$ \\
Set pressure & $p_{\text {set }}$ & $17 \mathrm{bar}$ \\
Coefficient of discharge & $C_{d}$ & 0.93 \\
Coefficient of restitution & $r$ & 0.8 \\
Maximum lift & $x_{\max }$ & $10 \mathrm{~mm}$ \\
Ambient temperature & $T_{0}$ & $293 \mathrm{~K}$ \\
Ambient pressure & $p_{0}$ & $1 \mathrm{bar}$ \\
Gas constant & $R$ & $288 \mathrm{~J} /(\mathrm{kg} \mathrm{K})$ \\
Specific heat ratio & $\kappa$ & 1.4 \\
\hline
\end{tabular}


the technique makes use of the conserved (Riemann) invariant quantities along the characteristic directions:

$$
a+\frac{\kappa-1}{2} v=\text { constant along } \frac{\mathrm{d} \xi}{\mathrm{d} t}=v+a
$$

and

$$
a-\frac{\kappa-1}{2} v=\text { constant along } \frac{\mathrm{d} \xi}{\mathrm{d} t}=v-a .
$$

(a)
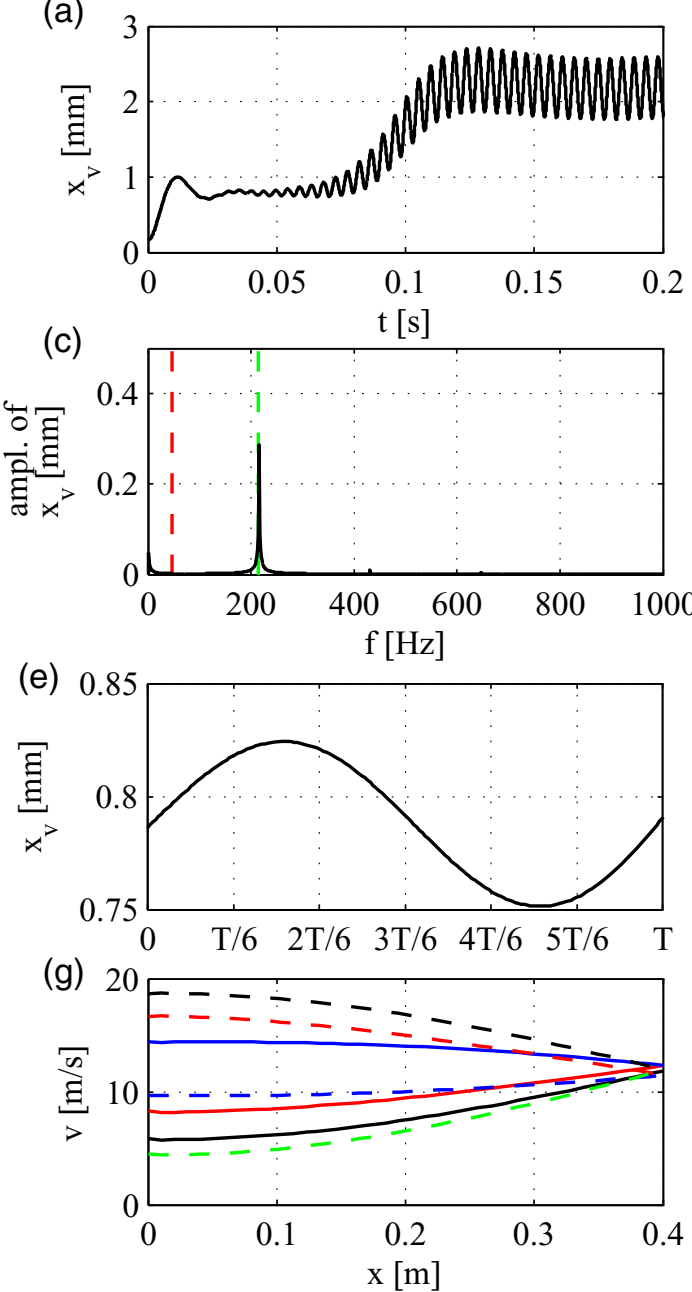

(b)

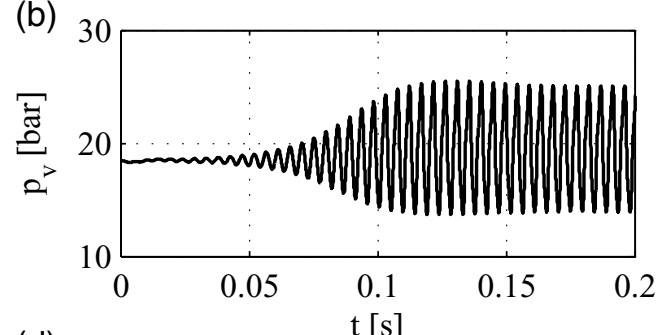

(d)
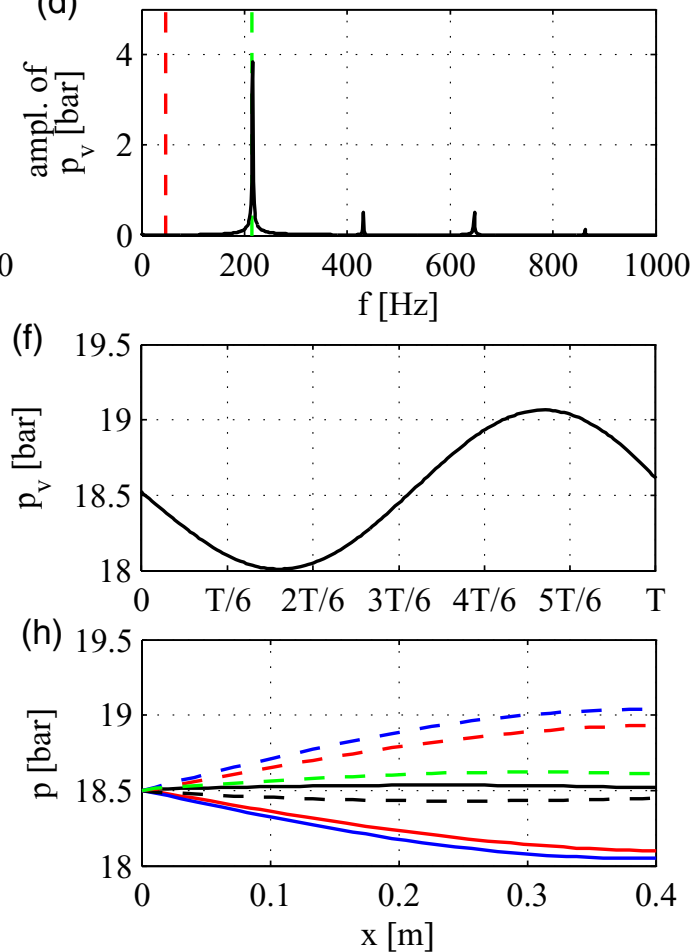

FIG. 2. An example run of the GDM with $L=0.4 \mathrm{~m}$ and $\dot{m}_{r \text {,in }}=0.5 \mathrm{~kg} / \mathrm{s}$ showing unstable valve motion. (a and b) The valve displacement and valve pressure $p_{v}=p(L, t)$ time histories; (c and d) the spectra of these time histories. Here dashed lines indicate the eigenfrequencies of the quarter wave (dashed line, close to $200 \mathrm{~Hz}$ ) and the valve (dashed line, closed to $46 \mathrm{~Hz}$ ); (e and f) one cycle of duration $T=0.04503 \mathrm{~s}$ between successive maxima of the valve displacement, starting at $t=0.0485 \mathrm{~s}$; ( $\mathrm{g}$ and h) the velocity and pressure distributions in the pipe at regular intervals $T / 6$ for this cycle, where similar line types (and colours, online) represent the same point in the cycle. 
Thus, at $\xi=0$ (the reservoir end of the pipe side), the 'isentropic inflow' boundary condition (13), the characteristic equation (16), the ideal gas law $(p=\rho R T)$ and the isentropic relations provide a sufficient number of equations to find the pressure, velocity, density and temperature. At the other $\xi=L$ end (the valve), we use (14) and (15).

\subsection{Parameter set and preliminary runs}

Unless otherwise stated, we use the parameter values defined in Table 2 in our GDM simulations. These represent typical values for a PRV with an air-like gas.

Figure 2 presents one of the computations for $L=0.4 \mathrm{~m}$ and $\dot{m}_{r \text {,in }}=0.5 \mathrm{~kg} / \mathrm{s}$. As can be seen in the valve displacement and valve pressure $\left(p_{v}=p(L, t)\right)$ time histories, in Fig. 2(a,b), respectively, the equilibrium is unstable. Figure $2(\mathrm{c}, \mathrm{d})$ represents the spectra of the time histories indicating that the evolving oscillation corresponds to the pipe: the dominant frequency of the oscillation coincides with the one-quarter of the pipe eigenfrequency. Figure 2(e,f) magnify one cycle of the unstable oscillation starting at $t=0.04541 \mathrm{~s}$ while Fig. $2(\mathrm{~g}, \mathrm{~h})$ shows the velocity and pressure distributions inside the pipe at the time instances indicated in Fig. 2(e,f).

It is clear from Fig. 2 and from plotting the frequency response, that the valve motion is governed by the pipe dynamics and both motions share the same dominant frequency being the quarter-wave frequency of the pipe. In other words, close to the onset of instability we experience a standing wave of both the velocity and pressure distribution within the pipe with a quarter of the pipe's wavelength.

\section{Reduced-order modelling: the QWM}

The pressure and velocity distributions in Fig. 2 show that the velocity at the valve end of the pipe $(\xi=$ $L)$ and the pressure at the reservoir side of the pipe $(\xi=0)$ are approximately constant. Moreover, the reservoir side of the pipe is fully open while the valve side of the pipe is almost closed due to the small valve openings. Thus, as was shown in Botros et al. (1997), we have the conditions to set up standing waves of wavelength $\left(n+\frac{1}{2}\right) / 2 L$, for $n=0,1,2, \ldots$ It is to be expected that for small amplitude motion (just beyond the onset of any instability) that most of the energy would lie in the leading order mode, which is indeed what we observed in Fig. 2(c,d). This observation allows the derivation of a reducedorder, simplified model that is likely to be valid for moderate amplitude motion close to the instability.

In accordance with our previous observations, we assume that the pressure and velocity distributions take the form

$$
p(\xi, t)=p_{r}(t)+B(t) \sin \left(2 \pi \frac{\xi}{4 L}\right)
$$

and

$$
v(\xi, t)=v_{L}(t)+C(t) \cos \left(2 \pi \frac{\xi}{4 L}\right)=\frac{1}{\sqrt{\rho_{e}} A} \zeta x(t) \sqrt{p_{r}(t)+B(t)}+C(t) \cos \left(2 \pi \frac{\xi}{4 L}\right) .
$$

The above ansatz ensures that the pressure at $\xi=0$ coincides with the reservoir pressure, which is a simplification of (13) as it neglects the pressure drop due to the acceleration of the fluid between the reservoir and the pipe entrance. At the valve end of the pipe, the pressure is $p_{v}(t)=p(L, t)=p_{r}(t)+B(t)$ with $B(t)$ being the amplitude of the pressure oscillations. Equation (18) ensures that the velocity profile satisfies the boundary condition (14) (with $v_{L}(t)$ being the gas velocity in the pipe at the valve end) with $C(t)$ being the amplitude of velocity oscillations in the pipe. Moreover, we make the following assumptions: 
- The temperature change inside the pipe is neglected, hence we have $p / \rho=$ const, which allows to compute the density merely based on the pressure, i.e. $\rho(p)$.

- We introduce the sonic velocity $a$ being $a^{2}=\mathrm{d} \rho / \mathrm{d} p=\kappa R T$ (see Zucrow \& Hoffman, 1976).

- With these assumptions, system (12) can be written in a simpler form:

$$
\begin{aligned}
\frac{\partial p}{\partial t}+v \frac{\partial p}{\partial \xi}+a^{2} \rho \frac{\partial v}{\partial \xi} & =0, \\
\frac{\partial v}{\partial t}+v \frac{\partial v}{\partial \xi} & =-\frac{1}{\rho} \frac{\partial p}{\partial \xi}+\lambda \frac{L}{D} v|v|,
\end{aligned}
$$

where-due to the assumption of constant temperature - the energy equation is no longer needed.

- Once the fluid compressibility was taken into account with the help of the sonic velocity, the density is kept constant, which is a standard approach in the computation of hydraulic transients (see, e.g. Wiley \& Streeter, 1978). In other words, once wave effects are included in the model via the sonic velocity, we neglect further changes in density.

- For a given inlet (driving) mass flow rate $\dot{m}_{\text {in }}$, the constant temperature and density values are evaluated at the equilibrium lift and pressure values. The density and sonic velocity values corresponding to the equilibrium will be denoted by $\rho_{e}$ and $a_{e}$, and, by virtue of the previous assumption, they are constants.

After making these simplifications, we substitute the assumed pressure and velocity distributions (17) and (18) into (19) and (20) and force them to be satisfied at the midpoint of the pipe, i.e. at $\xi=L / 2$. Performing this calculation and dividing both equations by $\sin \pi / 4=\cos \pi / 4=1 / \sqrt{2}$ gives

$$
\begin{aligned}
& \sqrt{2} \dot{p}_{r}+\dot{B}+\left(\sqrt{2} v_{L}+C\right) \frac{\pi}{2 L} B-a_{e}^{2} \rho_{e} \frac{\pi}{2 L} C=0, \\
& \sqrt{2} \dot{v}_{L}+\dot{C}-\left(\sqrt{2} v_{L}+C\right) \frac{\pi}{2 L} C=-\frac{1}{\rho_{e}} \frac{\pi}{2 L} B+\lambda \frac{L}{D}\left(\sqrt{2} \dot{v}_{L}+\dot{C}\right)\left|\sqrt{2} \dot{v}_{L}+\dot{C}\right| .
\end{aligned}
$$

Note that, for the ease of notation, we have used $v_{L}$ defined by (18) instead of the lengthy expression including $p_{r}(t)$ and $B(t)$. After some algebraic manipulation, and combining the valve and reservoir dynamics, we obtain the following set of five ODEs:

$$
\begin{aligned}
\dot{x}_{v}= & v_{v}, \\
\dot{v}_{v}= & \frac{A}{m}\left(p_{r}+B\right)-\frac{k}{m} v_{v}-\frac{s}{m}\left(x_{0}+x_{v}\right), \\
\dot{p}_{r}= & k_{1}\left(\dot{m}_{r, \text { in }}-\zeta x_{v} \sqrt{p_{r}+B}-k_{2} C\right), \\
\dot{B}= & -\sqrt{2} \dot{p}_{r}+k_{3} k_{4} C+\hat{\mathcal{F}}_{c e, 1}, \\
\dot{C}= & -\frac{1-\sqrt{2}}{\sqrt{2}} \frac{\zeta}{k_{2}} \frac{x_{v}}{\sqrt{p_{r}+B}} \dot{p}_{r}-\frac{1}{\sqrt{2}} \frac{k_{3} k_{4}}{k_{2}} \zeta \frac{x_{v} C}{\sqrt{p_{r}+B}} \\
& -\sqrt{2} \frac{\zeta}{k_{2}} v_{v} \sqrt{p_{r}+B}-\frac{k_{3}}{k_{4}} B+\hat{\mathcal{F}}_{c e, 2}+\hat{\mathcal{F}}_{e m, 2}+\hat{\mathcal{Q}}_{2},
\end{aligned}
$$


where

$$
\begin{aligned}
\hat{\mathcal{F}}_{c e, 1} & =-\frac{\pi}{2 \sqrt{2} L} B C-\frac{\pi \zeta}{2 A L \rho_{e}} x_{v} B \sqrt{p_{r}+B} \\
\hat{\mathcal{F}}_{c e, 2} & =\frac{\pi \zeta}{4 A L \rho_{e}} \frac{x_{v} B C}{\sqrt{p_{r}+B}}+\frac{\pi \zeta^{2}}{2 \sqrt{2} A^{2} L \rho_{e}^{2}} x_{v}^{2} B \\
\hat{\mathcal{F}}_{e m, 2} & =\frac{\pi}{2 \sqrt{2} L} C^{2}+\frac{\pi \zeta}{2 A L \rho_{e}} x_{v} C \sqrt{p_{r}+B} \\
\hat{\mathcal{Q}}_{2} & =-\frac{\lambda}{2 \sqrt{2} D}\left(C+\frac{\zeta \sqrt{2}}{k_{2}} x_{v} \sqrt{p_{r}+B}\right)\left|C+\frac{\zeta \sqrt{2}}{k_{2}} x_{v} \sqrt{p_{r}+B}\right|,
\end{aligned}
$$

with $k_{1}=a_{e}^{2} / V, k_{2}=\rho_{e} A, k_{3}=2 \pi\left(a_{e} / 4 L\right)$ and $k_{4}=\rho_{e} a_{e}$.

We shall refer to system (23-28) as the QWM. The terms $\hat{\mathcal{F}}_{c e, 1}, \hat{\mathcal{F}}_{c e, 2}$ and $\hat{\mathcal{F}}_{e m, 2}$ arise from the convective terms in the GDM $(v \partial p / \partial x$ and $v \partial v / \partial x)$, while $\mathcal{Q}_{2}$ results from the friction term $(\lambda L / D) v|v|$. We shall study the importance of these terms later in detail.

It is worth mentioning that the natural quarter-wave representation of the pipeline dynamics can be clearly seen if we fix the valve position at $x_{v}=0$ (closed valve) and assume constant reservoir pressure
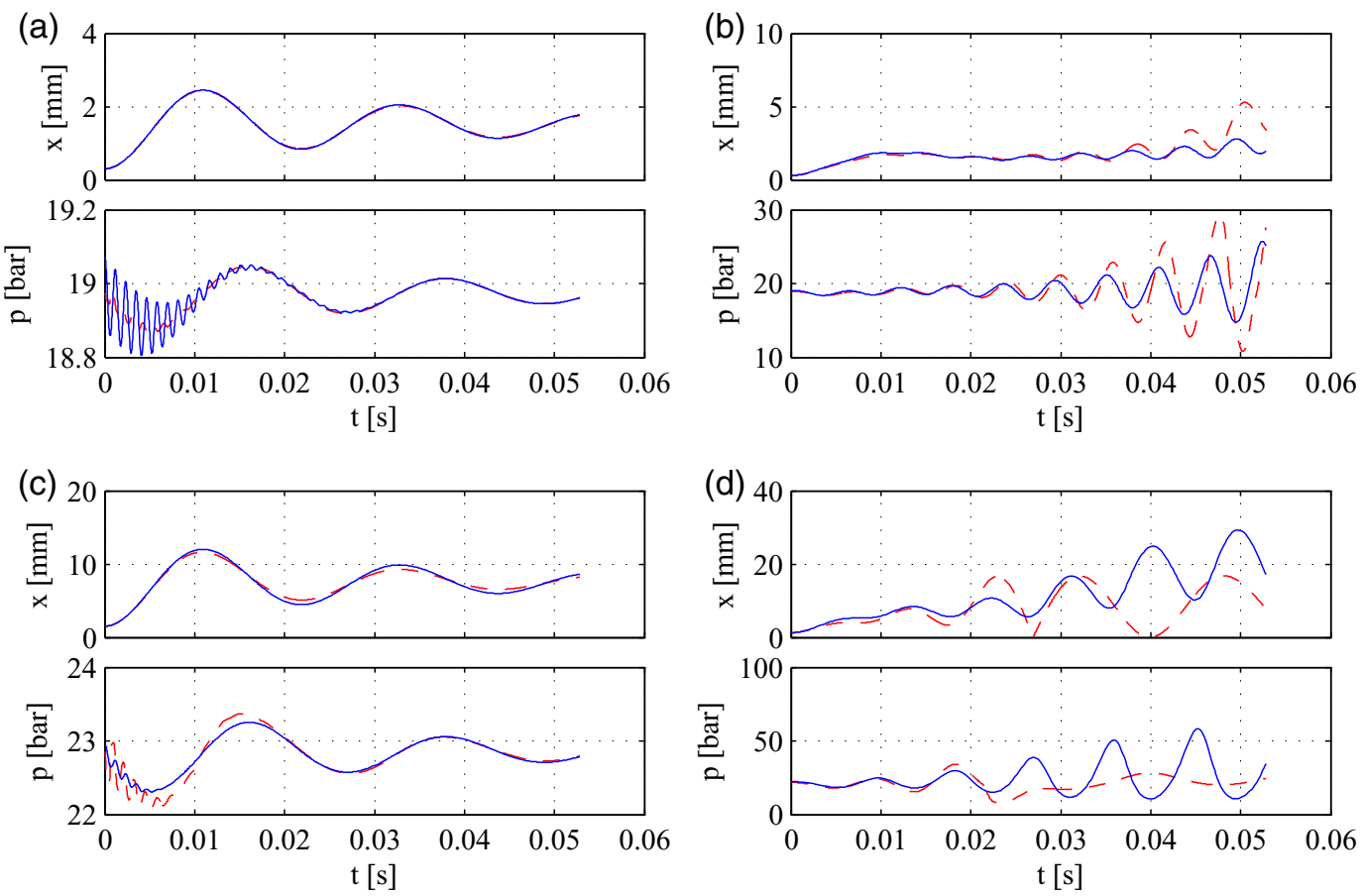

FIG. 3. Displacement and valve pressure time histories with (a) $\dot{m}_{r \text {,in }}=0.5 \mathrm{~kg} / \mathrm{s}$ and $L=0.1 \mathrm{~m}$, (b) $\dot{m}_{r \text {,in }}=0.5 \mathrm{~kg} / \mathrm{s}$ and $L=0.5 \mathrm{~m}$, (c) $\dot{m}_{r \text {,in }}=5 \mathrm{~kg} / \mathrm{s}$ and $L=0.1 \mathrm{~m}$ and (d) $\dot{m}_{r \text {,in }}=5 \mathrm{~kg} / \mathrm{s}$ and $L=0.8 \mathrm{~m}$. $p_{\text {set }}=17$ bar. The solid line (blue online) is from the GDM and the dashed line (red online) is from the QWM. 
$\dot{p}_{r}=0$. In this case, upon differentiating (28) once and using (26), we obtain

$$
\ddot{C}=-\frac{k_{3}}{k_{4}} \dot{B}=-k_{3}^{2} C=-\left(2 \pi \frac{a_{e}}{4 L}\right)^{2} C,
$$

which is a single degree-of-freedom oscillatory system with eigenfrequency $\omega=2 \pi\left(a_{e} / 4 L\right)=\omega_{\text {pipe }} / 4$.

\section{Comparison of the two models}

In this section, we compare the dynamic response of the GDM and the QWM.

\section{$4.1 \quad$ Numerical simulation}

First, we consider time histories of the two models for two different feed mass flow rates $\left(\dot{m}_{r, \text { in }}\right)$ and different pipe lengths. Figure 3 depicts the valve displacement and the valve pressure time histories at (a) $\dot{m}_{r \text {,in }}=0.5 \mathrm{~kg} / \mathrm{s}$ and $L=0.1 \mathrm{~m}$, (b) $\dot{m}_{r \text {,in }}=0.5 \mathrm{~kg} / \mathrm{s}$ and $L=0.5 \mathrm{~m}$, (c) $\dot{m}_{r \text {,in }}=5 \mathrm{~kg} / \mathrm{s}$ and $L=0.1 \mathrm{~m}$ and (d) $\dot{m}_{r \text {,in }}=5 \mathrm{~kg} / \mathrm{s}$ and $L=0.8 \mathrm{~m}$. In all cases, we take $p_{\text {set }}=17$ bar. The initial valve displacement is set to a somewhat lower position than its equilibrium value.

It is clearly seen in both models that the equilibrium is stable for short pipe lengths (see Fig. 3(a,c)). In this case, apart from a high-frequency initial transient, very good quantitative similarity is observed between the two models. In particular, the QWM predicts the frequency, phase and amplitude of the decaying oscillations of the GDM model. These oscillations represent damped quarter waves within the pipe. Presumably, the initial higher frequency transients in the GDM (most notable in Fig. 3(a)) are at least in part due to higher pipe harmonics, which are strongly damped for short pipes, whereas such higher frequency transients in the QWM can only be due to non-linear coupling between the valve and the quarter wave.

For longer pipes, as in Fig. 3(b,d), the motion does not settle down to equilibrium. Instead we observe an instability in the form of a self-excited oscillation. Note again that the close match between the two models while the oscillation amplitude remains moderate (within about 5 bar in $p$ ). However, as one might expect as it was only derived from a single mode expansion, the QWM loses its accuracy for larger amplitudes. This discrepancy is especially acute in the case of run (d), where the valve hits the seat and starts bouncing (which can be seen as the displacement $x$ approaching 0 ). The bouncing introduces sharp-gradient waves inside the pipe whose mode shapes are far from being harmonic, as assumed in (17) and (18). Hence, the QWM is likely to be quantitatively inaccurate beyond the first point of impact of the valve with its seat.

\subsection{Instability prediction}

We have shown that the equilibrium position of the valve can go unstable, which gives birth to large-amplitude oscillations even with bouncing valve motion. This phenomenon is well known in the relief valve literature (see Hayashi et al., 1997) and it is also common knowledge that the longer the pipeline between the valve and the reservoir is, the more unstable the valve becomes, see Funk (1964). In this section, we study the linear stability of the valve equilibrium with the help of the QWM, notably to uncover how the feed mass flow rate and the pipe length influence the valve motion close to the equilibrium. 

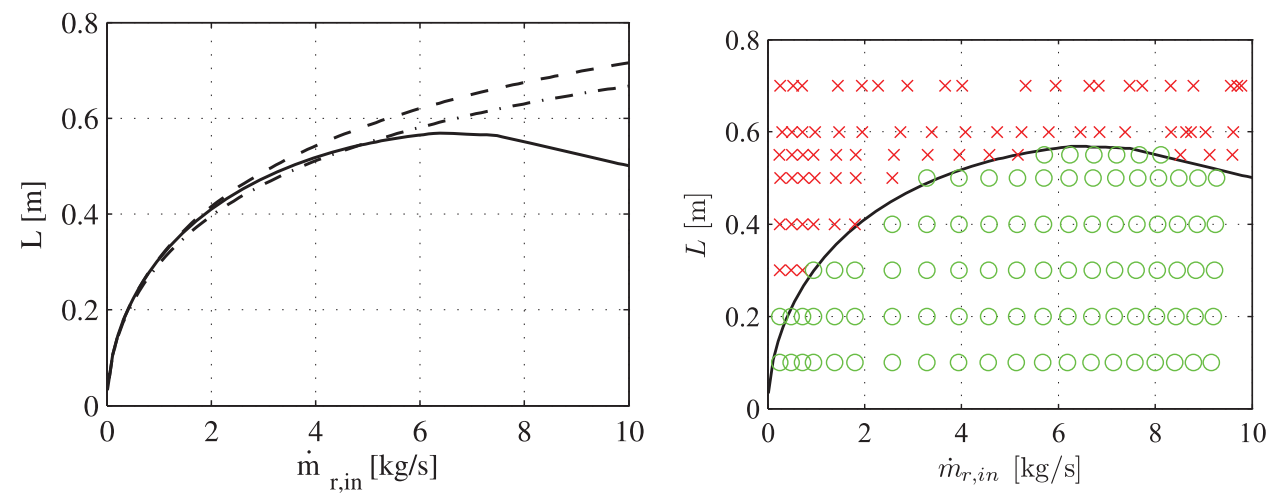

FIG. 4. Stability boundary of the QWM (solid line) obtained by linear stability analysis and comparison with other stability criteria; the system for short pipes (below the line) and unstable above for longer pipes. (Left): the dot-dashed line represents the same stability curve but for the QWM without both convective terms $\hat{\mathcal{F}}_{c e, 1}, \hat{\mathcal{F}}_{c e, 2}$ and $\hat{\mathcal{F}}_{e m, 2}$ and friction term $\mathcal{Q}_{2}$ set to zero. The dashed curve depicts the stability curve upon inclusion of the friction only, without the convective terms. (Right): the stability boundary compared with direct numerical simulation of the GDM, where instability is indicated by a (red online) cross and stability by a (green online) circle.

To reveal the linear stability of the system (23-27), we compute numerically its equilibrium and evaluate the Jacobian matrix, whose eigenvalues govern the local behaviour of the system. Instability occurs when a pair of imaginary eigenvalues cross the imaginary axis (a Hopf bifurcation). Using straightforward numerical continuation, we can compute a curve of such Hopf bifurcations as two parameters vary. These stability predictions can be compared with the results of simulation of the GDM. Here, we take as initial condition a $5 \%$ perturbation to the equilibrium valve lift $x_{e}$, keeping all other variables at their equilibrium values. Instability is deemed to occur whether the dynamics do not return to the equilibrium within $1 \mathrm{~s}$.

The results are shown in Fig. 4, using the pipe length $L$ and the feed flow rate $\dot{m}$ as primary parameters. In the right-hand panel of the stability boundary corresponding to the full QWM is depicted, together with the results of the GDM simulations. Circles represent parameter values for which the GDM predicts stability, while crosses represent unstable simulation runs. It is clearly seen that the QWM captures the stable and unstable regions not only qualitatively but also quantitatively. A single curve of instability is found, such that the valve is stable provided the pipe is sufficiently short. The critical pipe length for stability depends on the mass flow rate $\dot{m}$.

The left-hand plot shows the effects (using the QWM only) of friction and convective terms on the location of the instability. The results show that while these terms have negligible influence for small mass flow rates, this is definitely not the case for mass flow rates above $5 \mathrm{~kg} / \mathrm{s}$. As should be expected, inclusion of friction effects has a stabilizing influence. However, it is clear that the convective terms are destabilizing to the extent that the trend of the curve, for which the critical pipe length for instability increases with flow rate, is reversed and the interval of stable pipe lengths decreases as $\dot{m}$ increases above $6 \mathrm{~kg} / \mathrm{s}$. Note that this trend is also picked up in the results of the full GDM simulations shown in the right-hand panel.

\section{Conclusion}

The purpose of this paper has been the careful derivation of a reduced-order model for understanding fundamental instabilities due to pipe-valve interaction in direct spring PRVs. The key assumption is that 
at the point of onset of instability, the acoustic energy in the pipe is predominantly in the fundamental mode, a quarter wave. We have shown that, at least for the parameter values considered, pipe friction does not play a significant role. The convective terms are also not important for low flow rates, but in general introduce a destabilizing effect, which becomes more pronounced as the flow rate increases. The model so-derived can be augmented to capture more physics if necessary, for example, more complex valves and the effect of backpressure. Thus, it can allow a practitioner to perform rapid parametric analyses of stability thresholds for different potential system configurations.

In a companion paper (Bazsó et al., 2013), we conduct a thorough two-parameter bifurcation analysis of the QWM derived here. In particular, we use this model to investigate global dynamics caused by the interplay between the quarter-wave instability and the valve-only instability analysed in Hôs \& Champneys (2012).

\section{Funding}

Csaba Hös was partially supported by a Bolyai Scholarship of the Hungarian Academy of Sciences (Grant no. BO/00750/12).

\section{REFERENCES}

Askari, E., Jeong, K.-H. \& Amabili, M. (2013) Hydroelastic vibration of circular plates immersed in a liquid-filled container with free surface. J. Sound Vib, 332, 3064-3085.

Bazsó, C., Champneys, A. R. \& Hős, C. J. (2013) Bifurcation analysis of a simplified model of a pressure relief valve attached to a pipe. SIAM J. Appl. Dyn. Syst., 13, 704-721.

BAzsó, C. \& Hős, C. J. (2013) An experimental study on the stability of a direct spring loaded poppet relief valve. J. Fluids Struct., 42, 456-465.

Beune, A. (2009) Analysis of high-pressure safety valves. Ph.D. Thesis, Eindhoven University of Technology, Eindhoven, The Netherlands.

Botros, K. K., Dunn, G. H. \& Hrycyk, J. A. (1997) Riser-relief valve dynamic interactions. J. Fluids Eng., 119, 671-679.

Cebeci, T., Shao, J. P., Kafyeke, F. \& Laurendeau, E. (2005) Computational Fluid Dynamics for Engineers: From Panel to Navier-Stokes Methods with Computer Programs. Berlin: Springer.

Chabane, S., Plumejault, S., Pierrat, D., Couzinet, A. \& Bayart, M. (2009) Vibration and chattering of conventional safety relief valve under built up back pressure. Proceedings of the 3rd IAHR International Meeting of the WorkGroup on Cavitation and Dynamic Problems in Hydraulic Machinery and Systems. Paper presented at WorkGroup on Cavitation and Dynamic Problems in Hydraulic Machinery and Systems, Brno, Czech Republic, 14-16 October (P. Rudolf ed.). Brno: Brno University of Technology, pp. 281-294.

D’Netto, W. \& Weaver, D. S. (1987) Divergence and limit cycle oscillations in valves operating at small openings. J. Fluids Struct., 1, 3-18.

Francis, J. \& BetTs, P. L. (1998) Backpressure in a high-lift compensated pressure relief valve subject to single phase compressible flow. J. Loss Prevention Process Ind., 11, 55-66.

Funk, J. E. (1964) Poppet valve stability. J. Basic Eng., 86, 207.

Green, W. L. \& Woods, G. D. (1973) Some causes of chatter in direct acting spring loaded poppet valve. The 3rd International Fluid Power Symposium, Turin.

Habing, R. A. \& Peters, M. (2006) An experimental method for validating compressor valve vibration theory. J. Fluids Struct., 22, 683-697.

Hayashi, S. (1995) Instability of poppet valve circuit. JSME Int. J. Ser. C, Dyn. Control Robot. Des. Manuf., 38, 357-366.

Hayashi, S., Hayase, T. \& Kurahashi, T. (1997) Chaos in a hydraulic control valve. J. Fluids Struct., 11, 693-716. 
Hős, C. J. \& Champneys, A. R. (2012) Grazing bifurcations and chatter in a pressure relief valve model. Phys. D: Nonlinear Phenom., 241, 2068-2076.

KASAI, K. (1968) On the stability of a poppet valve with an elastic support: 1st report, considering the effect of the inlet piping system. Bull. JSME, 11, 1068-1083.

Khalak, A. \& Williamson, C. H. K. (1997) Fluid forces and dynamics of a hydroelastic structure with very low mass and damping. J. Fluids Struct., 11, 973-982.

Licsko, G., Champneys, A. R. \& Hős, C. J. (2009) Nonlinear analysis of a single stage pressure relief valve. Int. J. Appl. Math., 39, 12-26.

MacLeod, G. (1985) Safety valve dynamic instability: an analysis of chatter. J. Press. Vessel Technol., 107, $172-177$.

Madea, T. (1970a) Studies on the dynamic characteristic of a poppet valve: 1st report, theoretical analysis. Bull. JSME, 13, 281-289.

MadeA, T. (1970b) Studies on the dynamic characteristics of a poppet valve: 2 nd report, experimental analysis. Bull. JSME, 13, 290-297.

McCloy, D. \& McGuigan, R. H. (1964) Some static and dynamic characteristics of poppet valves. Proceedings of the Institution of Mechanical Engineers, vol. 179. Prof Eng Publishing, pp. 199-213.

MisRa, A., Behdinan, K. \& Cleghorn, W. L. (2002) Self-excited vibration of a control valve due to fluid-structure interaction. J. Fluids Struct., 16, 649-665.

Moussou, P., Gibert, R. J., Brasseur, G., Teygeman, C., Ferrari, J. \& Rit, J. F. (2010) Instability of pressure relief valves in water pipes. J. Press. Vessel Technol., 132, 041308.

Nayfeh, A. H. \& Bouguerra, H. (1990) Non-linear response of a fluid valve. Int. J. Non-Linear Mech., 25, 433-449.

SonG, X.-G., PARK, Y.-C. \& PARK, J.-H. (2013) Blowdown prediction of a conventional pressure relief valve with a simplified dynamic model. Math. Comput. Model., 57, 279-288.

Thomann, H. (1976) Oscillations of a simple valve connected to a pipe. Z. Angew. Math. Phys., 27, 23-40.

Vaughan, N. D., Johnston, D. N. \& EdGe, K. A. (1992) Numerical simulation of fluid flow in poppet valves. Proc. Inst. Mech. Eng., 206, 119-127.

VIEL, A. (2011) Strong coupling of modelica system-level models with detailed CFD models for transient simulation of hydraulic components in their surrounding environment. Proceedings 8th Modelica Conference, Paper presented at Modelica Association, Technical University, Dresden, Germany, 20-22 March (C. Clauß ed.). Linköping: Linköping University Electronic Press, pp. 256-265.

WARREN, M. D. (1983) Appropriate boundary conditions for the solution of the equations of unsteady onedimensional gas flow by the Lax-Wendroff method. Int. J. Heat Fluid Flow, 4, 53-59.

Wiley, E. B. \& Streeter, V. L. (1978) Fluid Transients, vol. 1. New York: McGraw-Hill International Book Co., $401 \mathrm{pp}$.

YE, Q. \& CHEN, J. (2009) Dynamic analysis of a pilot-operated two-stage solenoid valve used in pneumatic system. Simul. Modelling Pract. Theory, 17, 794-816.

Zucrow, M. J. \& Hoffman, J. D. (1976) Gas Dynamics, vol. 1. New York: John Wiley and Sons.

Zung, P. S. \& Perng, M. H. (2002) Nonlinear dynamic model of a two-stage pressure relief valve for designers. $J$. Dyn. Syst. Meas. Control, 124, 62-66. 